\title{
Extended meshfree methods without branch enrichment for cohesive cracks
}

\author{
Goangseup Zi · Timon Rabczuk · Wolfgang Wall
}

Received: 25 April 2006 / Accepted: 3 August 2006

(C) Springer-Verlag 2006

\begin{abstract}
An extended meshless method for both static and dynamic cohesive cracks is proposed. This new method does not need any crack tip enrichment to guarantee that the crack closes at the tip. All cracked domains of influence are enriched by only the sign function. The domain of influence which includes a crack tip is modified so that the crack tip is always positioned at its edge. The modification is only applied for the discontinuous displacement field and the continuous field is kept unchanged. In addition to the new method, the use of Lagrange multiplier is explored to achieve the same goal. The crack is extended beyond the actual crack tip so that the domains of influence containing the crack tip are completely cut. It is enforced that the crack opening displacement vanishes along the extension of the crack. These methods are successfully applied to several wellknown static and dynamic problems.
\end{abstract}

Keywords Extended element free Galerkin method (XEFG) - Local partition of unity - Cohesive cracks . Static and dynamic fractures

\section{Introduction}

If the tangent modulus of a material loses its positive slope, the strain gets localized to a narrow region.

G. Zi (凶)

Department of Civil and Environmental Engineering, Korea University, 5 Ga 1, An-Am Dong, Sung-Buk Gu, Seoul 136-701, South Korea

e-mail: g-zi@korea.ac.kr

T. Rabczuk · W. Wall

Institute for Numerical Mechanics,

Technical University of Munich,

Boltzmannstr. 15, 85748 Garching b. Munich, Germany
Eventually, the material separates as the displacement develops a discontinuity, i.e. a crack. It is well known that for rate-independent materials, the change of momentum equations due to the loss of hyperbolicity leads to a localization of deformation to a set of measure zero with zero energy dissipation [2]. So modeling the cracking process requires an additional model which dissipates energy during the crack initiation and growth in addition to the constitutive model if a softening rateindependent material is used.

In the cohesive crack model, traction on the crack surface is nonzero, depends on the crack opening displacement and thus, dissipates energy during the crack opening. Although there are numerous computational models in which the cohesive crack model is incorporated to model the fracture process, we may consider the most efficient methods as including meshfree methods [8,10,23], the inter-element method [14,35,50], and the extended finite element method (XFEM) [5,6, etc.]. Of course remeshing can be a solution, too but it is not practical because of the computational cost for remeshing and the inaccuracy caused by the projection between different meshes. In this paper, we discuss a new meshfree method based on the concept of the extended finite element method.

In classical meshfree methods [7,8,10,11,27,29,45], fracture problems are treated by the so called visibility criterion and its modifications. Here, the support is truncated by the crack $[7,8,11]$. Manipulation of the support near the crack may cause some difficulties. Meshfree methods, however, can provide better results for fracture problems than the classical finite element method thanks to high order interpolation and the smooth stress field. Impressive results using a multiscale cracking approach were shown by Liu et al. [28]. 
The recently developed XFEM has been proven to be an efficient tool for fracture problems. Unlike the classical finite element method, the solution space of XFEM includes the discontinuity caused by the crack. The method is based on the 'local' partition of unity, in which the solution space is enriched by a priori knowledge about the behavior of the solution near any discontinuity. Detailed discussions about the (local) partition of unity are found in the literature [e.g. 16,30]. Because only the nodes belonging to the elements cut by cracks are enriched, the number of additional degrees of freedom for the enrichment is minimized.

The only drawback of this method is the blending region inevitably introduced to the neighboring elements of the tip element in which the crack tip is positioned. Those elements in the blending region are partially enriched. The local partition of unity does not hold for them. Thus the solution becomes inaccurate in the region.

Ventura et al. [49] extended the concept of XFEM to the meshfree method for linear elastic cracks. Rabczuk and $\mathrm{Zi}$ et al. [40] developed the extended element-free Galerkin (XEFG) method for cohesive cracks. Like XFEM, the domains of influence cut by a crack are enriched by the step function and those including the crack tip by the branch function. XEFG has been applied to many static and dynamic fracture problems [40]. Because XEFG inherits the features of XFEM, there is the blending region near the crack tip. In this paper, we will develop a new XEFG to remove the blending region.

The paper is arranged as follows: The element free Galerkin method is briefly reviewed in Sect. 2. The developed enrichment is described in Sect. 3. In Sect. 4, the way of describing cracks is given. The governing equation of the continuum containing multiple cracks is given in Sect. 5. The variational principle and the discretized equation are given in Sect. 6. To verify the method, several examples are solved in Sect. 7. They are both static and dynamic problems. The conclusion for this work follows in Sect. 8.

\section{Element-free Galerkin (EFG) approximation}

An approximation of the displacement field $\boldsymbol{u}$ can be written as

$\boldsymbol{u}(\boldsymbol{X}, t)=\sum_{I \in \mathcal{W}} \Phi_{I}(\boldsymbol{X}) \boldsymbol{u}_{I}(t)$,

where $\Phi_{I}(\boldsymbol{X})$ is the shape function of particle $I$ at position $\boldsymbol{X}_{I}, \mathcal{W}$ is the set of all the particles and $\boldsymbol{u}_{I}$ are the parameters which scale the shape functions. In the EFG method [see e.g. 8,10,13,22], the shape functions are constructed as follows

$$
\begin{aligned}
& \Phi_{J}(\boldsymbol{X})=\boldsymbol{p}^{\mathrm{T}}(\boldsymbol{X}) \cdot \boldsymbol{A}(\boldsymbol{X})^{-1} \cdot \boldsymbol{D}\left(\boldsymbol{X}_{J}\right), \\
& \boldsymbol{A}(\boldsymbol{X})=\sum_{J} \boldsymbol{p}\left(\boldsymbol{X}_{J}\right) \boldsymbol{p}^{T}\left(\boldsymbol{X}_{J}\right) W\left(\bar{r}_{J} ; h_{J}\right), \\
& \boldsymbol{D}\left(\boldsymbol{X}_{J}\right)=\sum_{J} \boldsymbol{p}\left(\boldsymbol{X}_{J}\right) W\left(\bar{r}_{J} ; h_{J}\right) .
\end{aligned}
$$

Here, $\boldsymbol{p}$ are the base polynomials, $W$ is the kernel function, $\bar{r}_{J}=r / h_{J}$ is a dimensionless distance, $r=\left\|\boldsymbol{X}-\boldsymbol{X}_{J}\right\|$ is the distance from $\boldsymbol{X}_{J}$ to $\boldsymbol{X}$ and $h_{J}$ is the size of the domain of influence of particle $J$. The base polynomials are chosen such that $\boldsymbol{p}=(1, X, Y)$.

In addition to the fact that the order of continuity can be increased quite easily, meshfree methods have advantages over finite elements because of their smoothness and nonlocal interpolation character. Better stress distributions around the crack tip are expected, which must lead to a non-oscillatory crack propagation. Continuity in meshfree methods is governed by the continuity of the kernel function $W$. We used a cubic $B$-Spline as the kernel which is $C^{2}$, i.e.

$$
W(\bar{r} ; h)= \begin{cases}\frac{2}{3}-4 \bar{r}^{2}+4 \bar{r}^{3} & \text { for } \bar{r} \leq \frac{1}{2}, \\ \frac{4}{3}-4 \bar{r}+4 \bar{r}^{2}-\frac{4}{3} \bar{r}^{3} & \text { for } \frac{1}{2} \leq \bar{r} \leq 1, \\ 0 & \text { for } \bar{r}>1 .\end{cases}
$$

\section{Enrichment of displacement field}

\subsection{The enriched displacement field}

To model the discontinuity due to cracks, the approximation of Eq. (1) is enriched as follows

$$
\boldsymbol{u}(\boldsymbol{X}, t)=\boldsymbol{u}^{0}(\boldsymbol{X}, t)+\boldsymbol{u}^{e}(\boldsymbol{X}, t)
$$

where $\boldsymbol{u}^{0}$ is the continuous displacement field defined by (1) and $\boldsymbol{u}^{e}$ is the discontinuous (or the enriched) displacement field which is given by

$$
\boldsymbol{u}^{e}(\boldsymbol{X}, t)=\sum_{J \in \mathcal{E}} \boldsymbol{u}^{e, J}(\boldsymbol{X}, t)
$$

Here $\mathcal{E}$ is the set of all the cracks in the domain and $\boldsymbol{u}^{e, J}$ is the displacement enrichment by crack $J$. The displacement enrichment $\boldsymbol{u}^{e, J}$ is given by

$$
\boldsymbol{u}^{e, J}(\boldsymbol{X}, t)=\sum_{I \in \mathcal{W}_{J}} \Phi_{I}(\boldsymbol{X}) \Psi_{I}^{J}(\boldsymbol{X}) \boldsymbol{a}_{I}^{J}(t),
$$

where $\mathcal{W}_{J}$ is the set of the particles whose domain of influence is cut by crack $J, \Psi_{I}^{J}$ is the enrichment function for particle $I$ and crack $J$ and $\boldsymbol{a}_{I}$ are the additional degrees of freedom for the enrichment $\Psi_{I}^{J}$. Note that the shape function $\Phi_{I}$ in Eq. (8) does not have to be the same as $\Phi_{I}$ in Eq. (1) $[16,46,47]$. 
If a domain of influence is completely cut by a crack, it is enriched by using the sign function or the Heaviside function, which is now classical. We will use the sign function as the enrichment function here rather than use the Heaviside function because of its appealing symmetry. The sign function enrichment is equivalent to the Heaviside function enrichment. The enrichment is given by

$\Psi_{I}^{J}(\boldsymbol{X})=\operatorname{sign}\left[f^{J}(\boldsymbol{X})\right]-\operatorname{sign}\left[f^{J}\left(\boldsymbol{X}_{I}\right)\right]$.

Here $f^{J}(\boldsymbol{X})$ is the signed distance measured from $\boldsymbol{X}$ to crack $J$. It is defined as

$$
\begin{aligned}
f^{J}(\boldsymbol{X})= & \operatorname{sign}\left[\boldsymbol{n} \cdot\left(\boldsymbol{X}-\boldsymbol{X}^{J}\right)\right] \\
& \times \min \left\|\boldsymbol{X}-\boldsymbol{X}^{J}\right\| \quad \text { with } \boldsymbol{X}^{J} \in \Gamma_{c}^{J}
\end{aligned}
$$

in which $\boldsymbol{n}$ is the crack normal and $\Gamma_{c}^{J}$ represents crack $J$. The choice of the direction of the crack normal is completely arbitrary as long as it is consistent throughout the entire computation. Note that the minimum $\left\|\boldsymbol{X}-\boldsymbol{X}^{J}\right\|$ in Eq. (10) should be searched and determined first so that $\boldsymbol{X}^{J}$ is the closest point projection of $\boldsymbol{X}$ on $\Gamma_{c}^{J}$. Otherwise, $f^{J}(\boldsymbol{X})$ may not be accurate for a highly nonlinear crack. The sign function is defined as

$\operatorname{sign}(x)= \begin{cases}1 & \text { for } x>0 \\ -1 & \text { for } x<0 .\end{cases}$

There may be a case that a particle is sitting on a crack, which is not defined by Eqs. (9) and (11). Therefore an initial notch is placed between particles. The possibility of encountering such a case during crack propagation is very low, especially in a double precision computation; it never happened in the following example problems. If a crack grows to the position of a particle, the crack path may be little bit adjusted to preclude having such a case.

If the domain of influence is partially cut such as the case where the crack tip is located in a 'tip element', then the enrichment of Eq. (9) does not work. Then branch enrichment is needed [12,31]. If the cohesive crack is considered and a crack tip is located on an edge of a domain of influence, not inside of any domain of influence at the same time, a successful enrichment can be devised by using only the sign function or the Heaviside step function without other enrichment functions $[18,51]$.

The above idea can be applied to the extended finite element method only. The shape function for a node in the standard finite element method with $C^{0}$ approximation is completely decoupled from others except those for the neighbor nodes. Imagine a crack tip in a twodimensional finite element mesh; see Fig. 1a. The crack

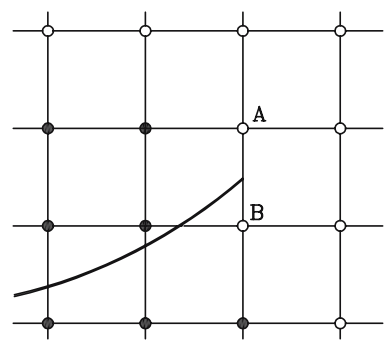

(a) finite element method

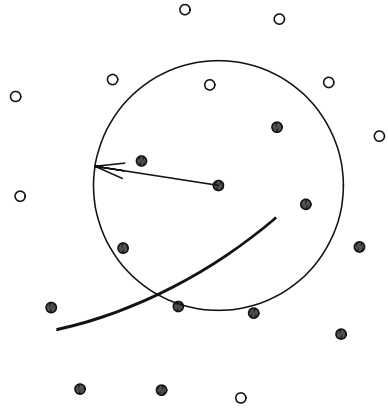

(b) meshfree method
Fig. 1 The enrichment for the crack tip by using the step function in $\mathbf{a}$ the finite element method and $\mathbf{b}$ meshfree methods; solids are enriched nodes and circles unenriched nodes

tip is positioned on the edge connecting nodes $A$ and $B$. Because the crack tip must close at the tip, i.e. the crack opening displacement at crack tip must be zero, the nodes $A$ and $B$ should not be enriched. Fig. $1 \mathrm{~b}$ is the case for meshfree methods. The domain of influence for a particle in the case of meshfree methods, is heavily overlapped with others. Therefore it is difficult to apply the idea to meshfree methods.

\subsection{Domain-decrease method}

$\mathrm{Zi}$ and Belytschko [51] proposed a simple idea to enrich triangular elements with only the sign function (11) without using the the branch enrichment even when the crack tip is located inside of an element, not on the edge. They modified the shape function of the discontinuous displacement field $\boldsymbol{u}^{e, J}$ in Eq. (8) so that the crack tip is always placed on the edge of the shape function in (8) as the crack grows. The modification is only for the tip element in which the crack tip is located. Once the crack tip grows into an element, this element, then, becomes the tip element. The shape function of the discontinuous displacement field for the tip element is modified as shown in Fig. 2. Because the shape function vanishes at its edge, the discontinuity from Eq. (8) must be zero beyond the edge. So branch enrichment is not needed. Of course the shape function of the continuous displacement $\boldsymbol{u}^{0}$ is completely independent of the crack growth.

We adopt their idea for meshfree methods, too. A crack is shown in Fig. 3. The domain of influence of particle 1 is completely cut by the crack. It is enriched by the sign function as in Eq. (9). The crack tip $P$ is located inside the domain of influence of particle 2 which is partially cut. As $\mathrm{Zi}$ and Belytschko [51] modified the shape function of triangular elements, we scale down the shape function $\Phi_{I}$ for the discontinuous displacement 

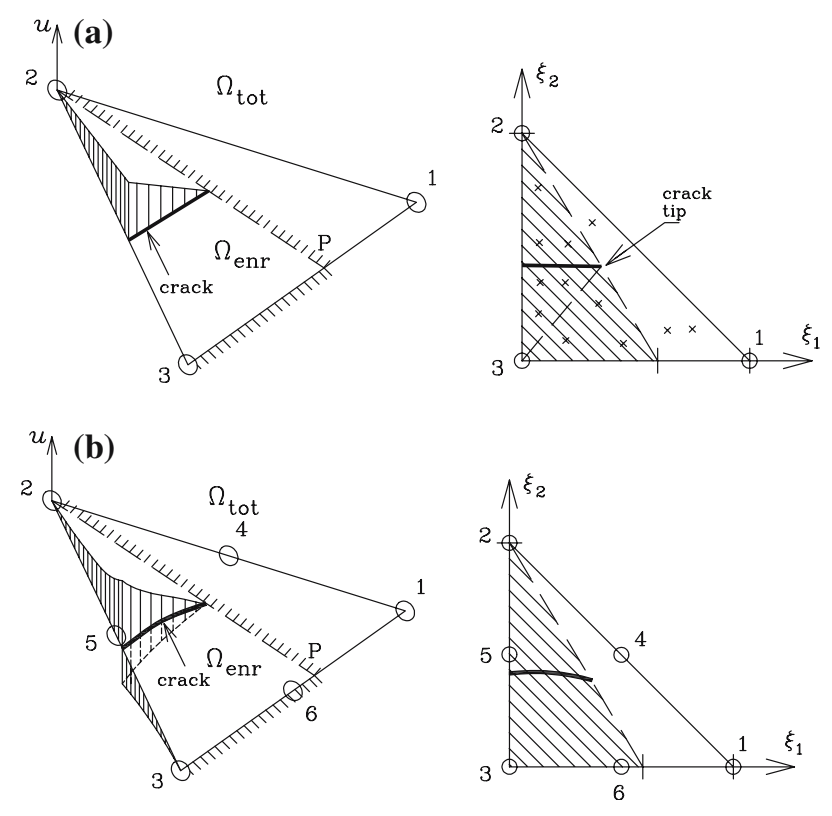

Fig. 2 The discontinuous displacement field by $\mathrm{Zi}$ and Belytschko [51]'s scheme in which the shape function for the enrichment is modified so that the crack tip is positioned at the edge; a three node triangular element and $\mathbf{b}$ six node triangular element

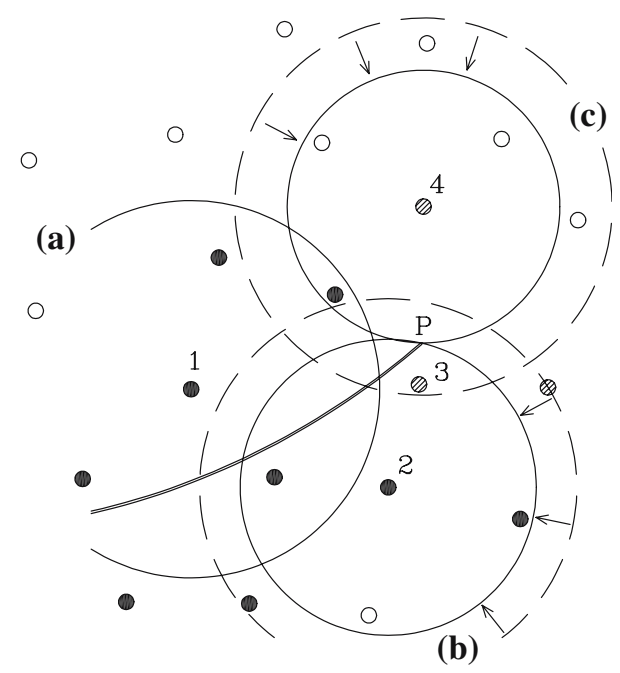

Fig. 3 Domain of influences a completely cut by the crack and $\mathbf{b}, \mathbf{c}$ partially cut; $\mathbf{b}$ is decreased so that the crack tip is positioned at its edge and $\mathbf{c}$ is not needed as its size is decreased; circles and hatched circles are unenriched particles, and solids enriched nodes

in Eq. (8). This is so that the crack tip is positioned at its edge as shown in the figure, i.e.

$$
\begin{aligned}
& \Phi_{J}^{*}(\boldsymbol{X})=\boldsymbol{p}^{T}(\boldsymbol{X}) \cdot \boldsymbol{A}^{*}(\boldsymbol{X})^{-1} \cdot \boldsymbol{D}^{*}\left(\boldsymbol{X}_{J}\right), \\
& \boldsymbol{A}^{*}(\boldsymbol{X})=\sum_{J} \boldsymbol{p}\left(\boldsymbol{X}_{J}\right) \boldsymbol{p}^{T}\left(\boldsymbol{X}_{J}\right) W\left(\bar{r}_{J}^{*} ; h_{J}^{*}\right), \\
& \boldsymbol{D}^{*}\left(\boldsymbol{X}_{J}\right)=\sum_{J} \boldsymbol{p}\left(\boldsymbol{X}_{J}\right) W\left(\bar{r}_{J}^{*} ; h_{J}^{*}\right)
\end{aligned}
$$

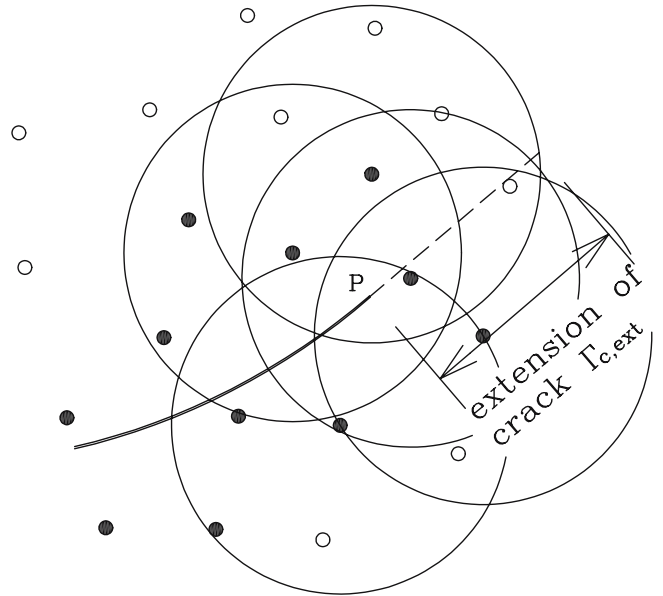

Fig. 4 The discontinuity $\Gamma_{\mathrm{c}, \text { ext }}$ beyond crack tip $P$ when particles are enriched by using only the sign function

in which the asterisk denotes the modification for the crack tip and $h^{*}$ is the modified size of the domain of influence. Note that the shape function for the continuous displacement field as in Eq. (1) is not changed.

The domain of influence of particle 3 is also partially cut. The shape function may be shrunk, too. However, we do not enrich the particle. Because the particle is very close to the crack tip, the shape function becomes very small compared to others after it is modified. The approximation for the discontinuous displacement field becomes bumpy. Therefore, when the domain of influence of a particle is partially cut, we enrich the particle if the support of the shape function includes at least one enriched particle after it is modified. Particle 4 is also not enriched because the shape function becomes not cut by the crack as it is modified.

One drawback of this method is that the crack appears to be shorter for particles close to the crack and the crack tip. This is because such particles are not enriched and hence do not feel the presence of the crack. We therefore expect the system response to be a little stiffer. However, we did not observe any severe difficulties or discrepancies compared to more accurate techniques as e.g. Lagrange multiplier method explained in the next section. As might become obvious, this method is well suited for adaptive procedures that will not only provide higher accuracy but also a better particle distribution around the crack tip

\subsection{Lagrange multiplier method}

Instead of modifying the shape function of the particle whose domain of influence is partially cut, we may 
consider the use of Lagrange multiplier method. If only the sign function enrichment of Eq. (9) is used, there is the extension of discontinuity $\Gamma_{\mathrm{c}, \text { ext }}$ beyond the crack tip; see Fig. 4. To model the actual crack, the discontinuity on $\Gamma_{\mathrm{c}, \text { ext }}$ should vanish. Because the condition should be satisfied along a line, not at a point, the Lagrange multiplier must be discretized too. To avoid introducing additional particles for the discretization, we use the same shape functions as those partially cut by the crack. The detailed formulation is given later.

\section{Description of cracks}

\subsection{The geometric information of cracks}

The crack $\Gamma_{c}$ is defined implicitly by the level set method. This level set method is a general tool for the description of evolving surfaces. It has been used for a wide range of applications [see 42]. It defines the surface of a discontinuity by a function of position $\boldsymbol{X}$ measured from the discontinuity. The function does not have to be the signed distance function defined in Eq. (10). For example, Ventura et al. [49] developed the vector level set method in which the distance of $\boldsymbol{X}$ to the discontinuity and its direction are used for the level set.

The level set with the signed distance function has successfully been incorporated in XFEM [6,32,48,51] and XEFG $[40,49]$. We will not explain the detailed crack tracing procedure with level sets in detail. Instead we refer the interested readers to the literature. However, we briefly describe how to treat crack branching and crack intersection which is different from the approach in Daux et al. [17]. The difference is that we do not use any special branch function in addition to the 'usual' enrichment.

Consider cracks shown in Fig. 5. Let $\mathcal{W}_{b}^{1}$ be the set of particles whose domain of influence is cut by the discontinuity $f^{1}(\boldsymbol{X})=0$ and $\mathcal{W}_{b}^{2}$ the corresponding set for $f^{2}(\boldsymbol{X})=0 . \mathcal{W}_{b}^{3}=\mathcal{W}_{b}^{1} \cap \mathcal{W}_{b}^{2}$. Zi et al. [53] proposed a computationally more efficient approach than Daux et al. [17]'s approach. $\mathrm{Zi}$ et al. modified the signed distance functions so that no cross terms are needed for junction or branch problems. By using the signed distance functions of the pre-existing and approaching crack, the signed distance function of the approaching crack is modified. Consider Fig. 5. Three different subdomains have to be considered: $\left(f^{1}<0, f^{2}<0\right),\left(f^{1}>0, f^{2}>0\right)$, $\left(f^{1}>0, f^{2}<0\right)$ as in Fig. 5b or $\left(f^{1}>0, f^{2}<0\right)$, $\left(f^{1}>0, f^{2}>0\right),\left(f^{1}<0, f^{2}<0\right)$ as in Fig. 5 d. The signed distance function of crack 1 of a point $\boldsymbol{X}$ is then obtained by:
$f^{1}(\boldsymbol{X})= \begin{cases}f_{0}^{1}(\boldsymbol{X}), & \text { if } f_{0}^{2}\left(\boldsymbol{X}^{(1)}\right) f_{0}^{2}(\boldsymbol{X})>0, \\ f_{0}^{2}(\boldsymbol{X}), & \text { if } f_{0}^{2}\left(\boldsymbol{X}^{(1)}\right) f_{0}^{2}(\boldsymbol{X})<0\end{cases}$

in which $f_{0}^{1}(\boldsymbol{X}), f_{0}^{2}(\boldsymbol{X})$ represent the signed distance functions of cracks 1 and 2 without consideration of the junction, respectively, and $\boldsymbol{X}^{(1)}$ is any point on crack 1 .

\subsection{Initiation and propagation of cracks}

We employed the loss of hyperbolicity criterion for crack initiation and propagation $[6,40]$. Therefore, a crack is initiated or propagated if the minimum eigenvalue of the acoustic tensor $\boldsymbol{Q}$ is smaller or equal to zero:

$\min \operatorname{eig}(\boldsymbol{Q}) \leq 0 \quad$ with $\boldsymbol{Q}=\boldsymbol{n} \cdot \boldsymbol{A} \cdot \boldsymbol{n}$,

where $\boldsymbol{n}=\left[\begin{array}{lll}\cos \theta & \sin \theta\end{array}\right]$ is the normal to the crack surface depending on the angle $\theta, \boldsymbol{A}=\boldsymbol{C}^{t}+\boldsymbol{\sigma} \otimes \boldsymbol{I}, \boldsymbol{\sigma}$ is the stress tensor and $\boldsymbol{C}^{t}$ is the fourth order tangential modulus.

For a rate-independent material, loss of hyperbolicity serves as a criterion for crack initiation. In the case of a rate-dependent material, the transition criterion to discontinuum is governed by the same condition which, however, is called material stability, rather than loss of hyperbolicity, since the equations are regularized. For a Rankine material, a crack is initiated when the principal tensile stress exceeds the tensile strength. The crack is then normal to the direction of the principal tensile stress. Note that the Rankine criterion is a special case of the loss of hyperbolicity condition for mode $I$ fracture.

Assuming that the loss of hyperbolicity condition should be satisfied at the moving crack tip with a certain velocity at any time, Belytschko et al. [6] proposed an approach to calculate the speed of crack propagation. Because the continuum transits to the discontinuum right at the crack tip, the eigenvalue obtained from the localization analysis (16) must be equal to zero at all the active crack tips, i.e.

$\frac{\mathrm{D} e}{\mathrm{D} t}=0$

in which $e=\boldsymbol{h} \cdot \boldsymbol{Q} \cdot \boldsymbol{h}$ is the hyperbolicity indicator and $\boldsymbol{h}$ is the eigenvector from the localization analysis in (16). Using the transport theorem, one can change (17) to

$\frac{\partial e}{\partial t}+\boldsymbol{v}^{c} \cdot \nabla e=0 \quad$ with $\boldsymbol{v}^{c}=v^{c} \boldsymbol{s}$,

where $v^{c}$ is the speed of crack propagation, $s$ is the propagation direction which fulfills the condition of $\boldsymbol{n} \cdot \boldsymbol{s}=0$. 
Fig. 5 The change of the signed distance function when one crack joins to another (a)

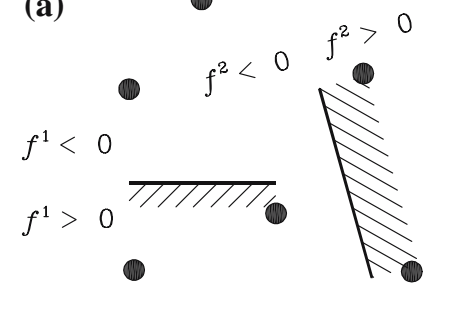

(c)

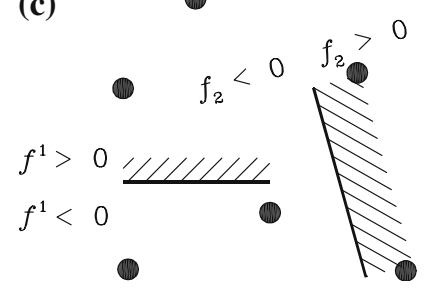

(b)

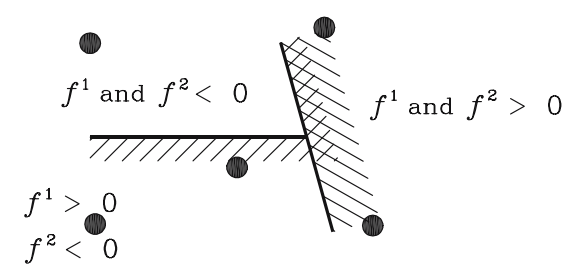

(d)
4.3 Measure of crack opening displacement

The jump in the displacement is governed only by the enrichment and is given by

$\llbracket \boldsymbol{u}(\boldsymbol{X}) \rrbracket=2 \sum_{J \in \mathcal{E}} \sum_{I \in W_{J}(\boldsymbol{X})} \Phi_{I}^{J}(\boldsymbol{X}) \boldsymbol{a}_{I}^{J}$

The normal part $\delta_{n}$, i.e. the crack opening and the tangential part $\delta_{t}$, the crack sliding are given by

$\delta_{n}=\boldsymbol{n} \cdot \llbracket \boldsymbol{u}(\boldsymbol{X}) \rrbracket$,

$\delta_{t}=\left\|\llbracket \boldsymbol{u}(\boldsymbol{X}) \rrbracket-\boldsymbol{n} \delta_{n}\right\|$.

More details are given in [12]. If not mentioned otherwise, we only consider normal tractions to the crack surface and neglect any mode $I I$ effect.

\section{Governing equations}

5.1 The momentum equation and the boundary conditions

The strong form of the momentum equation in the total Lagrangian description is given by

$\varrho_{0} \ddot{\boldsymbol{u}}=\nabla_{0} \cdot \boldsymbol{P}+\varrho_{0} \boldsymbol{b} \quad$ in $\quad \Omega_{0} \backslash \Gamma_{0}^{c}$

with boundary conditions:

$\boldsymbol{u}(\boldsymbol{X}, t)=\overline{\boldsymbol{u}}(\boldsymbol{X}, t) \quad$ on $\Gamma_{0}^{u}$,

$\boldsymbol{n}^{0} \cdot \boldsymbol{P}(\boldsymbol{X}, t)=\overline{\boldsymbol{t}}(\boldsymbol{X}, t) \quad$ on $\Gamma_{0}^{t}$,

$\boldsymbol{n}_{0} \cdot \boldsymbol{P}^{-}=\boldsymbol{n}_{0} \cdot \boldsymbol{P}^{+}=\boldsymbol{t}_{c 0}$ on $\Gamma_{0}^{c}$,

$\boldsymbol{t}_{c 0}=\boldsymbol{t}_{c 0}(\llbracket \boldsymbol{u} \rrbracket) \quad$ on $\Gamma_{0}^{c}$,

where $\varrho_{0}$ is the initial mass density, $\ddot{\boldsymbol{u}}$ is the acceleration, $\boldsymbol{P}$ denotes the nominal stress tensor, $\boldsymbol{b}$ designates the body force, $\overline{\boldsymbol{u}}$ and $\overline{\boldsymbol{t}}$ are the prescribed displacement and traction, respectively, $\boldsymbol{n}_{0}$ is the outward normal to the domain and $\Gamma_{0}^{u} \cup \Gamma_{0}^{t} \cup \Gamma_{0}^{c}=\Gamma_{0},\left(\Gamma_{0}^{u} \cap \Gamma_{0}^{t}\right) \cup\left(\Gamma_{0}^{t} \cap \Gamma_{0}^{c}\right) \cup$ $\left(\Gamma_{0}^{c} \cap \Gamma_{0}^{u}\right)=\emptyset$. Moreover, we assume that the stresses $\boldsymbol{P}$ at crack surface $\Gamma_{0}^{c}$ are bounded. Since the stresses are not well defined in the crack, the crack surface $\Gamma_{0}^{c}$ is excluded from the domain $\Omega_{0}$ which is considered as an open set.

\subsection{Constitutive equations}

We use Rankine type materials and the Lemaitre damage model [26]. For the Lemaitre model, the stressstrain behavior is

$\boldsymbol{\sigma}=(1-D) \boldsymbol{C}: \boldsymbol{\varepsilon}$,

where $\boldsymbol{C}$ is the elasticity tensor and $D$ is a scalar damage variable which ranges from 0 to a maximum of 1 . The damage evolution depends on the effective strain $\bar{\epsilon}$ :

$$
\begin{aligned}
D(\bar{\epsilon})= & 1-(1-A) \epsilon_{D_{0}} \bar{\epsilon}^{-1} \\
& -A \exp \left[-B\left(\bar{\epsilon}-\epsilon_{D_{0}}\right)\right]
\end{aligned}
$$

with

$\bar{\epsilon}=\sqrt{\sum_{i=1}^{3} \epsilon_{i}^{2} \mathcal{H}\left(\epsilon_{i}\right)}$,

where $A, B$ and $\epsilon_{D_{0}}$ are the material parameters and $\epsilon_{i}$ are the principal strains and with

$\mathcal{H}(x)= \begin{cases}1 & \text { if } x>0, \\ 0 & \text { if } x<0\end{cases}$

\subsection{Cohesive cracks}

In the cohesive crack model, there is no stress singularity near the crack tip. The traction on the crack surfaces is a function of the crack opening displacement. The 
cohesive crack models that are most popularly used in practice are shown in Fig. 6. For problem simplicity, we use the linear cohesive law and the exponential law in this study. Various kinds of the bilinear cohesive law can be found in Bažant and $\mathrm{Zi}$ [4]. The cohesive traction $\tau$ for the linear law is given by

$\tau(\delta)= \begin{cases}\tau_{f}\left(1-\frac{\delta}{\delta_{c}}\right) & \text { if } \delta \leq \delta_{c}, \\ 0 & \text { if } \delta>\delta_{c}\end{cases}$

where $\delta$ is the crack opening displacement, $\delta_{c}$ is the critical crack opening displacement beyond which the cohesive traction is reduced to zero and $\tau_{f}$ is the strength of the material. The exponential cohesive law is given by

$\tau(\delta)= \begin{cases}\tau_{f} \exp \left(-\frac{\tau_{f}}{G_{f}} \delta\right) & \text { if } \delta \leq \delta_{c}, \\ 0 & \text { if } \delta>\delta_{c} .\end{cases}$

If a potential for the cohesive crack is defined, the unidirectional relation of Eq. (31) can be extended to general mixed mode problems, too $[3,14,36]$. Here we consider only the mode $I$ crack separation. The computational implementation of Eq. (31) including the unloading and reloading behaviors can be found in the literature [e.g. 6].

\section{Discretized equations}

6.1 The discrete governing equations

The weak form of the momentum equation is given by

$\delta W=\delta W_{\text {int }}+\delta W_{\text {kin }}-\delta W_{\text {ext }}-\delta W_{\text {coh }}$,

where $\delta W_{\text {int }}, \delta W_{\text {kin }}, \delta W_{\text {ext }}, \delta W_{\text {coh }}$ are the virtual works by the internal stress, the inertia force, the external

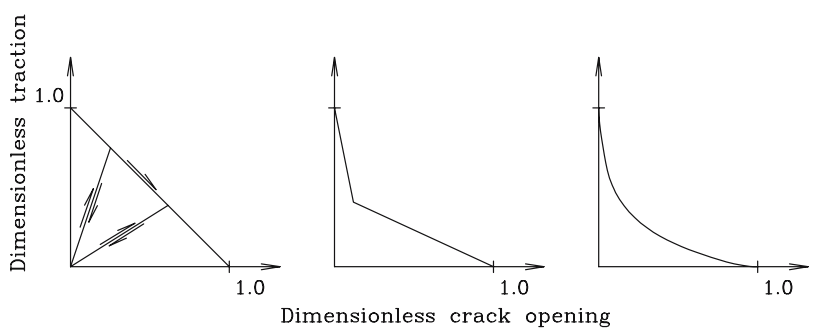

Fig. 6 The types of the cohesive laws frequently used in practice; a linear (or triangular), b bilinear and exponential cohesive laws traction and the cohesive traction, respectively. They are given by

$$
\begin{aligned}
& \delta W_{\text {int }}=\int_{\Omega_{0} \backslash \Gamma_{0}^{c}}(\nabla \otimes \delta \boldsymbol{u})^{T}: \boldsymbol{P} \mathrm{d} \Omega, \\
& \delta W_{\mathrm{kin}}=\int_{\Omega_{0} \backslash \Gamma_{0}^{c}} \varrho_{0} \delta \boldsymbol{u} \cdot \ddot{\boldsymbol{u}} \mathrm{d} \Omega, \\
& \delta W_{\mathrm{ext}}=\int_{\Omega_{0} \backslash \Gamma_{0}^{c}} \varrho_{0} \delta \boldsymbol{u} \cdot \boldsymbol{b} \mathrm{d} \Omega_{0}+\int_{\Gamma_{0}^{t}} \delta \boldsymbol{u} \cdot \overline{\boldsymbol{t}}_{0} \mathrm{~d} \Gamma, \\
& \delta W_{\mathrm{coh}}=\int_{\Gamma_{0}^{c}} \llbracket \delta \boldsymbol{u} \rrbracket \cdot \boldsymbol{\tau} \mathrm{d} \Gamma .
\end{aligned}
$$

Substituting the continuous and discontinuous displacement fields $\boldsymbol{u}^{0}$ and $\boldsymbol{u}^{e}$ in Eqs. (1) and (7), and the crack opening displacement $\llbracket \boldsymbol{u} \rrbracket$ in Eq. (19) to the weak form, we obtain

$$
\begin{aligned}
& \delta W_{\mathrm{int}}=\sum_{I \in \mathcal{W}} \delta \boldsymbol{u}_{I}^{T} \int_{\Omega_{0} \backslash \Gamma_{0}^{c}} \nabla_{0} \Phi_{I}(\boldsymbol{X})^{T}: \boldsymbol{P} \mathrm{d} \Omega \\
& +\sum_{J \in \mathcal{E}} \sum_{K \in \mathcal{W}_{J}} \delta \boldsymbol{a}_{K}^{J} \int_{\Omega_{0} \backslash \Gamma_{0}^{c}} \nabla_{0} \\
& \times\left[\Phi_{K}(\boldsymbol{X}) \Psi_{K}^{J}(\boldsymbol{X})\right]^{T}: \boldsymbol{P} \mathrm{d} \Omega, \\
& \delta W_{\text {kin }}=\sum_{I \in \mathcal{W}} \delta \boldsymbol{u}_{I}^{T} \sum_{J \in \mathcal{W}_{\Omega_{0} \backslash \Gamma_{0}^{c}}} \varrho_{0} \Phi_{I}(\boldsymbol{X})^{T} \cdot \Phi_{J}(\boldsymbol{X}) \mathrm{d} \Omega \ddot{\boldsymbol{u}}_{J} \\
& +\sum_{I \in \mathcal{W}} \delta \boldsymbol{u}_{I}^{T} \sum_{J \in \mathcal{E}} \sum_{K \in \mathcal{W}_{J}} \\
& \times \int_{\Omega_{0} \backslash \Gamma_{0}^{c}} \varrho_{0} \Phi_{I}(\boldsymbol{X})^{T} \cdot \Phi_{K}(\boldsymbol{X}) \Psi_{K}^{J}(\boldsymbol{X}) \mathrm{d} \Omega \ddot{\boldsymbol{a}}_{J}^{K} \\
& +\sum_{J \in \mathcal{E}} \sum_{K \in \mathcal{W}_{J}} \delta \boldsymbol{a}_{K}^{J} \sum_{I \in \mathcal{W}_{\Omega_{0} \backslash \Gamma_{0}^{c}}} \varrho_{0} \\
& \times\left[\Phi_{K}(\boldsymbol{X}) \Psi_{K}^{J}(\boldsymbol{X})\right]^{T} \cdot \Phi_{I}(\boldsymbol{X}) \mathrm{d} \Omega \ddot{\boldsymbol{u}}_{I} \\
& +\sum_{J \in \mathcal{E}} \sum_{K \in \mathcal{W}_{J}} \delta \boldsymbol{a}_{I}^{K^{T}} \sum_{L \in \mathcal{E}} \sum_{M \in \mathcal{W}_{L} \Omega_{0} \backslash \Gamma_{0}^{c}} \varrho_{0} \\
& \times\left[\Phi_{K}(\boldsymbol{X}) \Psi_{K}^{J}(\boldsymbol{X})\right]^{T} . \\
& \Phi_{M}(\boldsymbol{X}) \Psi_{M}^{L}(\boldsymbol{X}) \mathrm{d} \Omega \ddot{\boldsymbol{a}}_{M}^{L},
\end{aligned}
$$




$$
\begin{aligned}
\delta W_{\mathrm{ext}}= & \sum_{I \in \mathcal{W}} \delta \boldsymbol{u}_{I}^{T} \int_{\Omega_{0} \backslash \Gamma_{0}^{c}} \varrho_{0} \Phi_{I}(\boldsymbol{X})^{T} \cdot \boldsymbol{b} \mathrm{d} \Omega \\
& +\sum_{I \in \mathcal{W}} \delta \boldsymbol{u}_{I}^{T} \int_{\Gamma_{0}^{t}} \Phi_{I}(\boldsymbol{X})^{T} \cdot \overline{\boldsymbol{t}}_{0} \mathrm{~d} \Gamma \\
\delta W_{\mathrm{coh}}= & 2 \sum_{J \in \mathcal{E}} \sum_{K \in \mathcal{W}_{J}} \delta \boldsymbol{a}_{K}^{T} \int_{\Gamma_{0}^{c}} \Phi_{K}(\boldsymbol{X})^{T} \cdot \overline{\boldsymbol{t}}_{c} \mathrm{~d} \Gamma .
\end{aligned}
$$

Using the fundamental lemma of the variational principle, one obtains the discretized equation, i.e.

$\mathbf{M} \ddot{\mathbf{q}}=\mathbf{f}_{\mathrm{ext}}+\mathbf{f}_{\mathrm{coh}}-\mathbf{f}_{\mathrm{int}}$,

where $\mathbf{M}$ is the consistent mass matrix, $\mathbf{q}$ is the generalized parameters, $\mathbf{f}_{\text {ext }}, \mathbf{f}_{\text {int }}, \mathbf{f}_{\text {coh }}$ are the discrete external, internal and cohesive force vectors, respectively. The expressions for $\mathbf{M}, \mathbf{q}, \mathbf{f}_{\mathrm{ext}}, \mathbf{f}_{\mathrm{coh}}$ and $\mathbf{f}_{\mathrm{coh}}$ are given by

$$
\begin{aligned}
& \mathbf{M}=\int_{\Omega_{0} \backslash \Gamma_{0}^{c}} \varrho_{0}\left[\begin{array}{cccc}
\Phi^{0^{T}} & \Phi^{0} & \Phi^{0^{T}} & \Phi^{e} \\
& & & \\
\Phi^{e T} & \Phi^{0} & \Phi^{e T} & \Phi^{0}
\end{array}\right] \mathrm{d} \Omega, \\
& \mathbf{f}_{\text {int }}=\int_{\Omega_{0} \backslash \Gamma_{0}^{c}} \mathbf{B}^{0^{T}} \boldsymbol{P} \mathrm{d} \Omega+\int_{\Omega_{0} \backslash \Gamma_{0}^{c}} \mathbf{B}^{e T} \boldsymbol{P} \mathrm{d} \Omega, \\
& \mathbf{f}_{\mathrm{ext}}=\int_{\Omega_{0} \backslash \Gamma_{0}^{c}} \varrho_{0} \Phi^{0^{T}} \boldsymbol{b} \mathrm{d} \Omega+\int_{\Gamma_{0}^{t}} \Phi^{0^{T}} \overline{\boldsymbol{t}}_{0} \mathrm{~d} \Gamma, \\
& \mathbf{f}_{\mathrm{coh}}=2 \int_{\Gamma_{0}^{c}} \Phi^{T} \boldsymbol{t}_{c 0} \mathrm{~d} \Gamma \\
& \mathbf{q}=\left\{\begin{array}{l}
\mathbf{u} \\
\mathbf{a}
\end{array}\right\} \\
& \mathbf{u}=\left[\boldsymbol{u}_{I}\right]^{T} \quad \forall I \in \mathcal{W} \text { and } \mathbf{a}=\left[\boldsymbol{a}_{K}^{J}\right]^{T} \\
& \forall K \in \mathcal{W}_{J}, \forall J \in \mathcal{E}, \\
& \Phi^{0}=\left[\boldsymbol{\Phi}_{I}\right] \quad \forall I \in \mathcal{W} \text { and } \Phi^{e}=\left[\boldsymbol{\Phi}_{J} \boldsymbol{\Psi}_{J}^{K}\right] \\
& \forall K \in \mathcal{W}_{J}, \forall J \in \mathcal{E}, \\
& \mathbf{B}^{0}=\nabla_{0} \Phi^{0} \text { and } \mathbf{B}^{e}=\nabla_{0} \Phi^{e} \text {. }
\end{aligned}
$$

If the Lagrange multiplier is used, Eq. (33) should be modified as follows:

$\delta W_{\mathrm{L}}=\delta W+\delta(\boldsymbol{\Lambda} \cdot \llbracket \boldsymbol{u} \rrbracket)$

in which $\delta W_{\mathrm{L}}$ is the general variation with constraint and $\boldsymbol{\Lambda}$ is the Lagrange multiplier vector. As mentioned in Sect 3.3, the Lagrange multiplier is defined for $\Gamma_{\mathrm{c} \text {,ext }}$ and is interpolated using the shape function of the meshfree method. Therefore the discretized Lagrange multiplier is given by

$\Lambda=\Phi^{0} \lambda$, where $\lambda$ are the vector parameters to interpolate the Lagrange multiplier $\boldsymbol{\Lambda}$. Through the standard procedure for deriving the discrete equations [e.g. 9], we obtain

$$
\begin{aligned}
& \mathbf{M} \ddot{\mathbf{q}}=\mathbf{f}_{\mathrm{ext}}+\mathbf{f}_{\mathrm{coh}}-\mathbf{f}_{\mathrm{int}}-\mathbf{f}_{\mathrm{con}}, \\
& \mathbf{G} \mathbf{a}=\mathbf{0} .
\end{aligned}
$$

Here $\mathbf{f}_{\text {con }}$ is the extra force term due to the constraint and is given by

$\mathbf{f}_{\mathrm{con}}=\lambda^{T} \mathbf{G}$

in which $\mathbf{G}$ is

$\mathbf{G}=2 \Phi^{0^{T}} \Phi^{e}$.

\subsection{Numerical integration}

To integrate the discrete Eqs. (43) to (46), the integration cells, consisting of rectangles, are used. The integration cell without any crack is integrated by the standard Gauss quadrature. The cells with cracks are subdivided into many triangles where the edges are aligned with the cracks. These cells may be integrated by the same standard procedure. This integration scheme is now classical and the detailed information can be found in the literature [e.g. 12,40,51].

If there is a stress singularity near the crack tip, the above procedure cannot be used and care should be taken. Recently, a simple technique has been developed to integrate the singularity accurately [25]. In the cohesive crack model, however, there is no such a singularity. The classical triangulation produces a satisfactory result.

\section{Numerical examples and discussions}

\subsection{Arrea-Ingraffea beam}

The first example is the Arrea and Ingraffea [1] beam. The beam was loaded as shown in Fig. 7. The initial elastic modulus was $28,000 \mathrm{MPa}$, tensile strength was $2.8 \mathrm{MPa}$, Poisson's ration $v=0.18$ and the fracture energy was $G_{\mathrm{f}}=100 \mathrm{~N} / \mathrm{m}$. The beam failed because of a mixed tensile-shear failure.

We used the Lemaitre's damage model in tension for the continuum [e.g. 26], and loss of hyperbolicity for crack initiation. A linear decaying cohesive law was used. The concrete was assumed to be linear elastic in compression. The beam was discretized with approximately 2,700, 6,000 and 40,000 particles with a structured uniform particle arrangement. With a refinement where the crack was expected, even lower discretizations would be possible. 


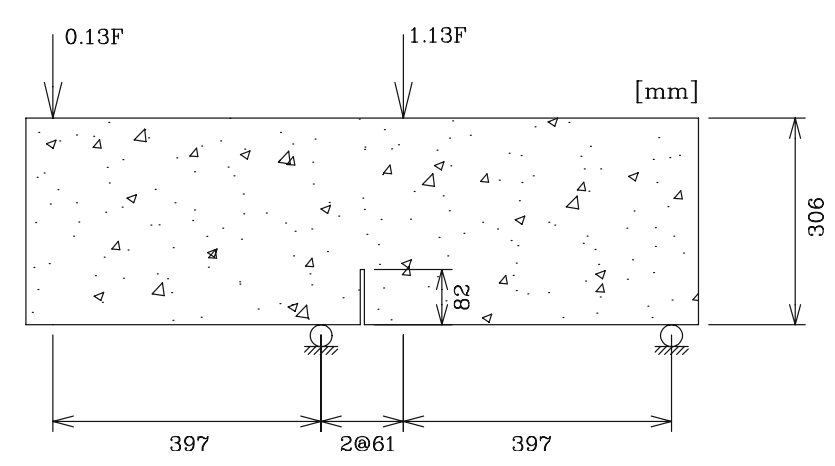

Fig. 7 The tensile/shear beam from Arrea and Ingraffea [1]

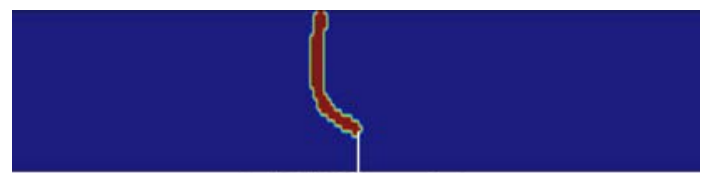

(a) 2,700 particles

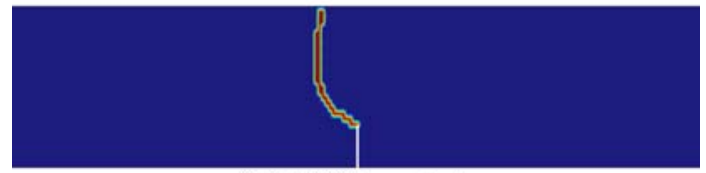

(b) 6,000 particles

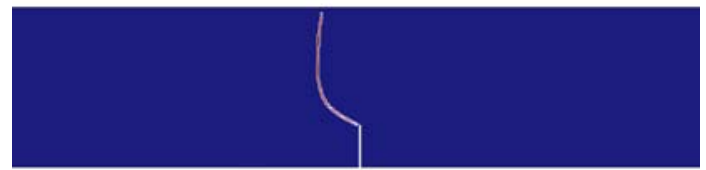

(c) 40,000 particles

Fig. 8 The crack pattern of the Arrea-Ingraffea beam for the domain-decrease method with different number of particles

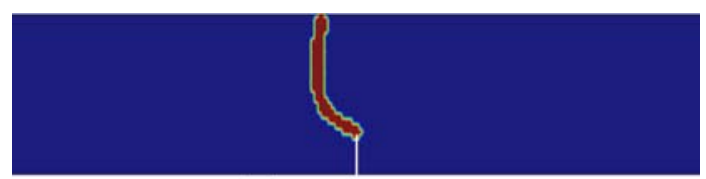

(a) 2,700 particles

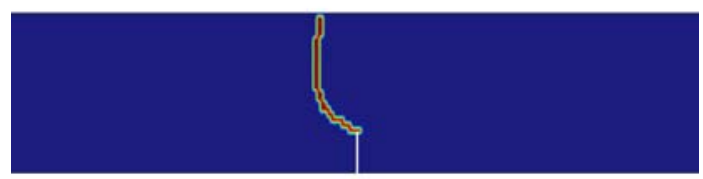

(b) 6,000 particles

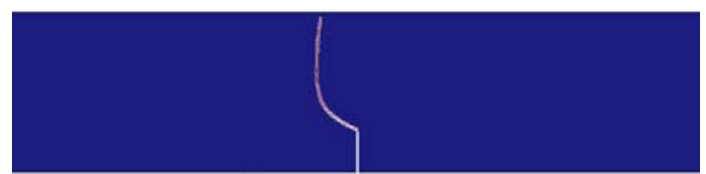

(c) 40,000 particles

Fig. 9 The crack pattern of the Arrea-Ingraffea beam for the Lagrange multiplier method with different number of particles
The crack paths for different refinements are shown in Fig. 8 for the domain-decrease method and Fig. 9 for the Lagrange multiplier method. The curvature of the cracks obtained from both methods was similar to the one from the experiment. The differences between the two methods were very marginal. The load displacement curve (right of the notch) is shown in Fig. 10. It lies in the experimental scatter.

7.2 Double edge-notched specimen with curved cracks

Consider a double edge-notched specimen as shown in Fig. 11. Experimental data is available in NooruMohamed [33]. At first, the specimen was loaded in shear without any vertical force. At a certain load, the type of loading was then changed. In the second stage, the shear force was kept constant and the displacement in the vertical direction was gradually increased. There were two distinct cracking patterns by the change of the shear force at $P_{\mathrm{s}}=5 \mathrm{kN}$ and $P_{\mathrm{s}}=27.5 \mathrm{kN}$. In the first case, the crack path was pretty straight. For the second case, a highly curved crack pattern was observed. This cracking pattern was because of the high shear force and not surprising. In addition to the experiments, several authors have carried out numerical studies of this experiment [see e.g. 34,37].

The material properties, according to [33], were compressive strength $f_{\mathrm{c}}=46 \mathrm{MPa}$, splitting tensile strength $f_{\mathrm{s}}=3.78 \mathrm{MPa}$ that yields according to the CEB-FIP Model Code [e.g. 20] a tensile strength of $f_{\mathrm{t}}=0.8 f_{s}$, initial Young's modulus $E=29 \mathrm{GPa}$, Poisson's ratio $v=0.22$ and fracture energy $G_{\mathrm{f}}=110 \mathrm{~J} / \mathrm{m}^{2}$.

For the first case in which load reversion is at $P_{\mathrm{s}}=$ $5 \mathrm{kN}$, the crack pattern is shown in Fig. 12 at two different steps. Figs. 12a,b present the results from the domaindecrease method; Figs. 12c,d present the results from the Lagrange multiplier method. Though the domaindecrease method is much simpler, the results are almost identical. A crack propagated almost perpendicular to the vertical direction since the shear force was very small. When the two cracks were 'overlapping' each other, the upper crack still propagated while the lower crack is arrested because of unloading of the ligament next to the crack. The results for the other load reversion, $P_{\mathrm{s}}=27.5 \mathrm{kN}$, are shown in Fig. 13 for different load steps. As in the experiment, the large shear force wa sufficient to produce a highly curved crack. The simulation was able to predict this behavior well. The load deflection curve is shown in Fig. 14 and agrees well with the experimental results. For the higher shear force load, the computed load deflection curve overestimated the experimental one. However, this overestimation was reported by several authors, e.g. Patzák and Jirásek [37]. 


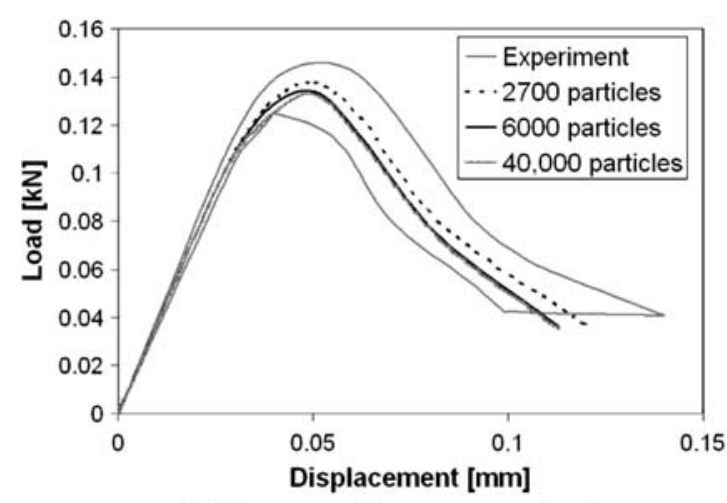

(a) Domain-decrease method

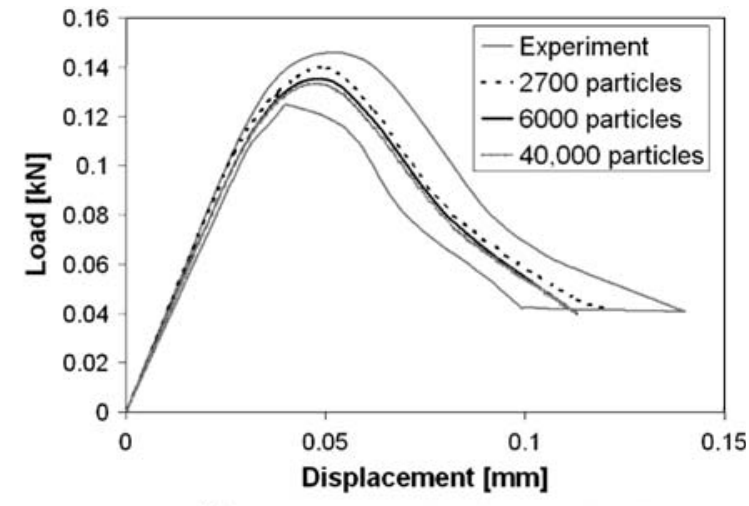

(b) Lagrange multiplier method

Fig. 10 The load deflection curve of the Arrea-Ingraffea beam for different numbers of particles

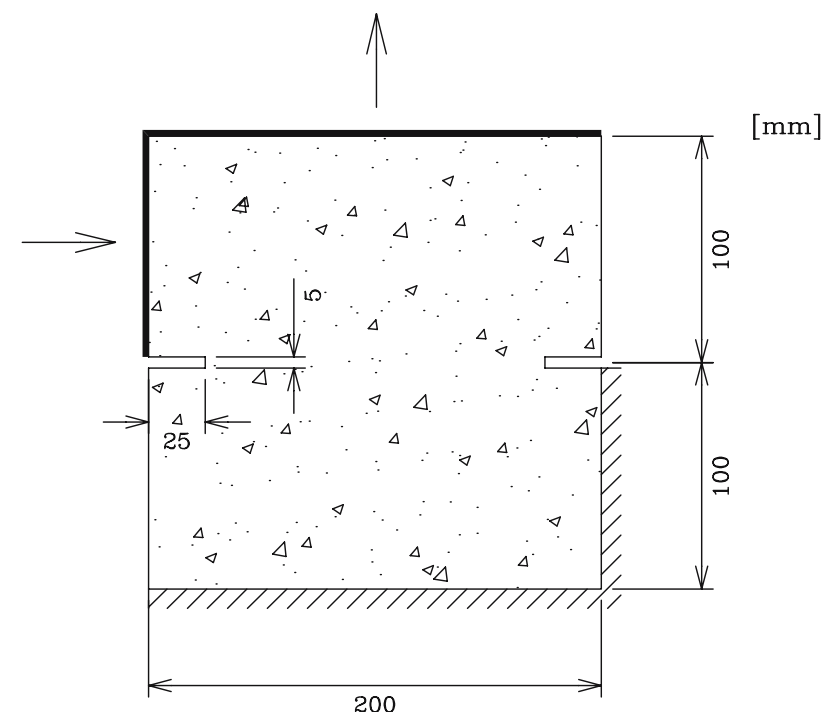

Fig. 11 Nooru-Mohamed's double edge-notched specimen [33]

Our results agreed well with the numerical results in [37]. The domain-decrease method gave little bit stiffer results compared to the Lagrange multiplier method. This is probably because of the slight underestimation of the crack length for the mixed mode problem with multiple loading paths.

\subsection{Cracks in dams}

Carpinteri et al. [15] and Shi [44] studied the behavior of concrete gravity dams subjected to hydraulic loading. The test set-up is shown in Fig. 15. The experimental concrete material parameters were measured to Young's modulus $E=35.7 \mathrm{GPa}$, Poisson's ratio $v=0.1$, density $\varrho=2,400 \mathrm{~kg} / \mathrm{m}^{3}$, tensile strength $f_{\mathrm{t}}=3.5 \mathrm{MPa}$ and fracture energy $G_{\mathrm{f}}=0.184 \mathrm{~N} / \mathrm{mm}$. We carried out sim- ulations with two different refinements and used 12,000 and 25,000 particles. The final crack pattern is shown in Fig. 16 for the domain decrease method; the results for the Lagrange multiplier method looks almost identical.

\subsection{Crack branching}

In the following, we examined the performance of these methods in a crack branching problem. We considered a rectangular prenotched specimen as shown in Fig. 17. The length of the rectangle was $0.1 \mathrm{~m}$ and the width $0.04 \mathrm{~m}$. Plane strain conditions were assumed. Initially, there was a horizontal crack from the left edge to the center of the plate over the entire thickness. A tensile traction of $1 \mathrm{MPa}$ was applied on the top and bottom edges.

We used the Lemaitre's damage law [26], loss of hyperbolicity and an exponential decaying cohesive law (Fig. 6c. The material constants were $\varrho=2,450 \mathrm{~kg} / \mathrm{m}^{3}$, $E=32 \mathrm{MPa}, v=0.2$ and $A=1.0, B=7,300$ and $\epsilon_{D_{0}}=$ $8.5 \times 10^{-5}$ for the Lemaitre model. Two dimensional computations of this problem have previously been reported by $\mathrm{Xu}$ and Needleman [50], Falk et al. [19], Belytschko et al. [6], Rabczuk and Belytschko et al. [38]. A three dimensional computation was made by Rabczuk and Belytschko [39]. Experimental data is available, see Ravi-Chandar [41], Sharon and Fineberg [43], Fineberg et al. [21].

The crack pattern is shown in Fig. 18 for different computations. It does not show mesh dependence. On the left hand side of Fig. 18 are the results of the coarse discretization and on the right hand side the results of the fine discretization. Two crack branches occurred similar to the results obtained by Rabczuk and Belytschko $[38,39]$. 
Fig. 12 The crack pattern of the double edge-notch specimen for load reversion of $P_{\mathrm{s}}=5 \mathrm{kN}$ at different load steps; $\mathbf{a}, \mathbf{b}$ for domain-decrease method; c,d for Lagrange multiplier method

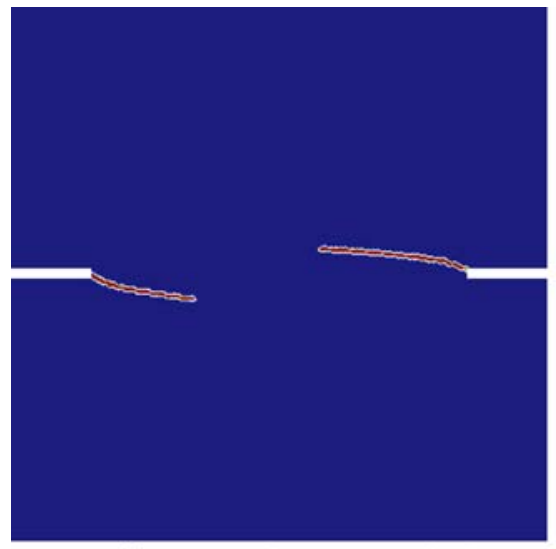

(a) Domain-decrease method

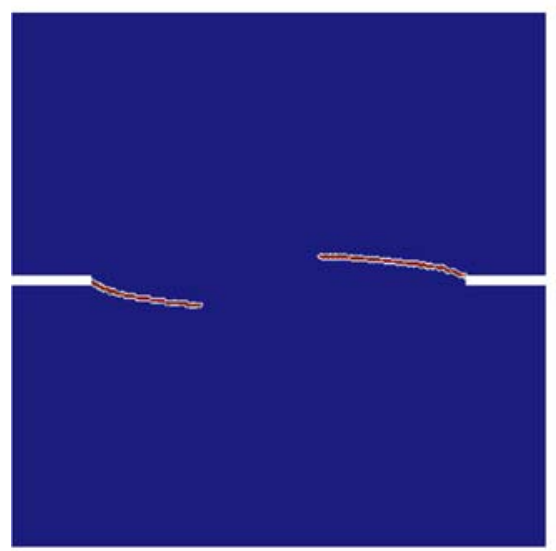

(c) Lagrange multiplier method

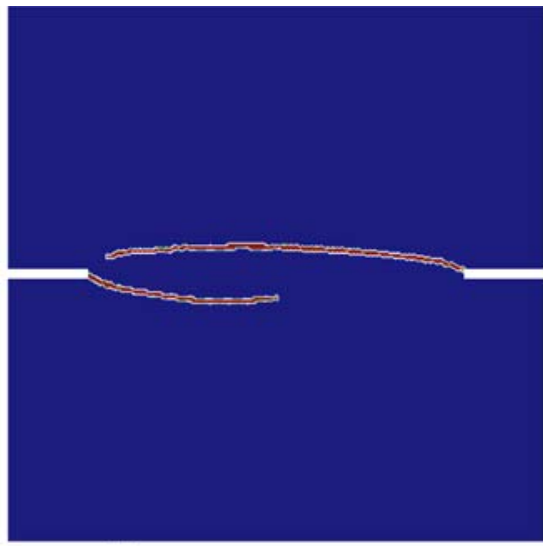

(b) Domain-decrease method

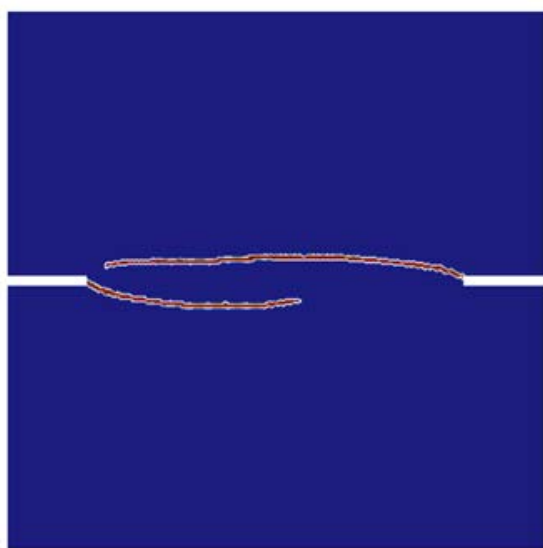

(d) Lagrange multiplier method
The time history of the crack speed is shown in Fig. 19. The crack starts to propagate at about $0.012 \mathrm{~ms}$. As expected, the crack speed was highest at the time of crack branching. At the first branching, the crack speed almost reached the theoretical Rayleigh wave speed. Afterwards, the crack speed decreased. The crack speed of the upper branch is shown in the figure. The crack speed of the lower branch is very similar as Fig. 18 might indicate.

\subsection{John and Shah's beam}

John and Shah [24] performed a series of dynamic experiments on notched concrete beams. Figure 20 shows the test setup. They varied the load rate and the location of the notch. The location of the notch is defined by a parameter $\gamma=l_{1} / l_{2}$ in which $l_{1}, l_{2}$ are the distances to the notch and the left support from the center, respectively; see the figure. The parameter $\gamma$ ranges from 0.5 to 0.77 .
The rate of loading ranged from a slow strain rate of $10^{-6} / s$ for the quasi-static experiments to a dynamic load with strain rates of $0.5 / s$. Two different failure modes were observed in the experiments. The first one was a pure mode $I$ failure in the middle of the beam; the second one was a mixed mode failure where the crack started to propagate from the notch. The transition from the mode $I$ to the mixed mode failure depended on the location of the notch and differed for the dynamic and the static loading conditions. For the same location of the notch, the slopes of the crack (for the mixed mode failure) for the quasi-static and dynamic loading were almost equal. We studied here the dynamic loading. If the notch was at $\gamma=0.77$, a mixed mode failure occurred in the dynamic experiment (a crack evolved from the notch and another crack was initiated in the middle of the specimen). The load was applied via a boundary velocity condition given by John and Shah [24].

First, we focused on the notched beam number 4 $(\gamma=0.77)$ under dynamic loading. We now present the results for the domain-decrease method. We tested 
Fig. 13 The crack pattern of the double edge-notch specimen for load reversion of $P_{\mathrm{s}}=17.5 \mathrm{kN}$ at different load steps; a-c for

domain-decrease method; d for Lagrange multiplier method

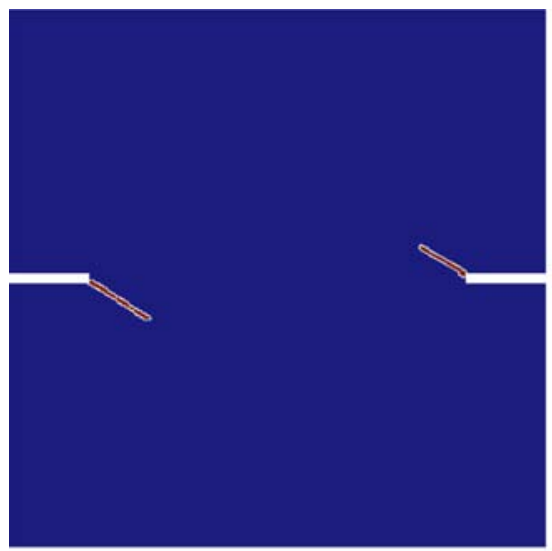

(a) Domain-decrease method

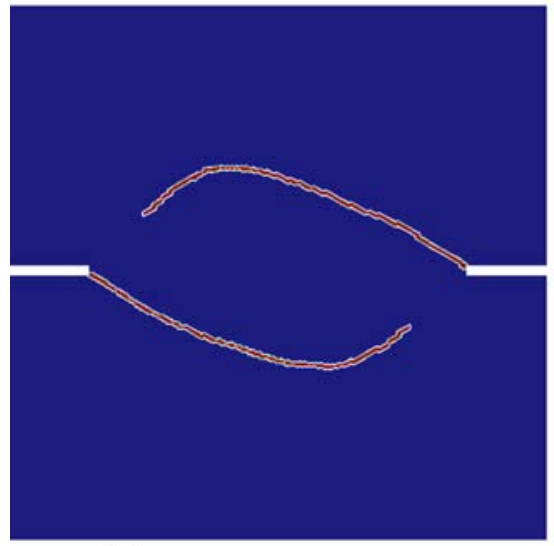

(c) Domain-decrease method

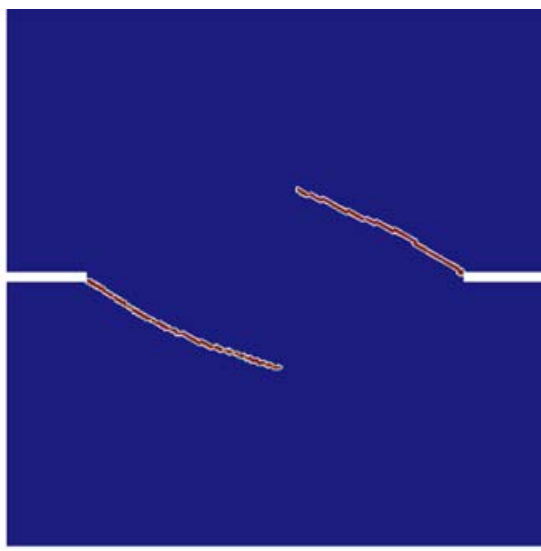

(b) Domain-decrease method

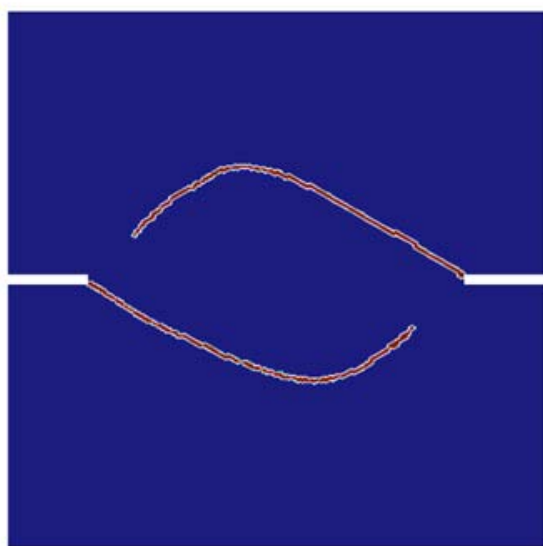

(d) Lagrange multiplier method
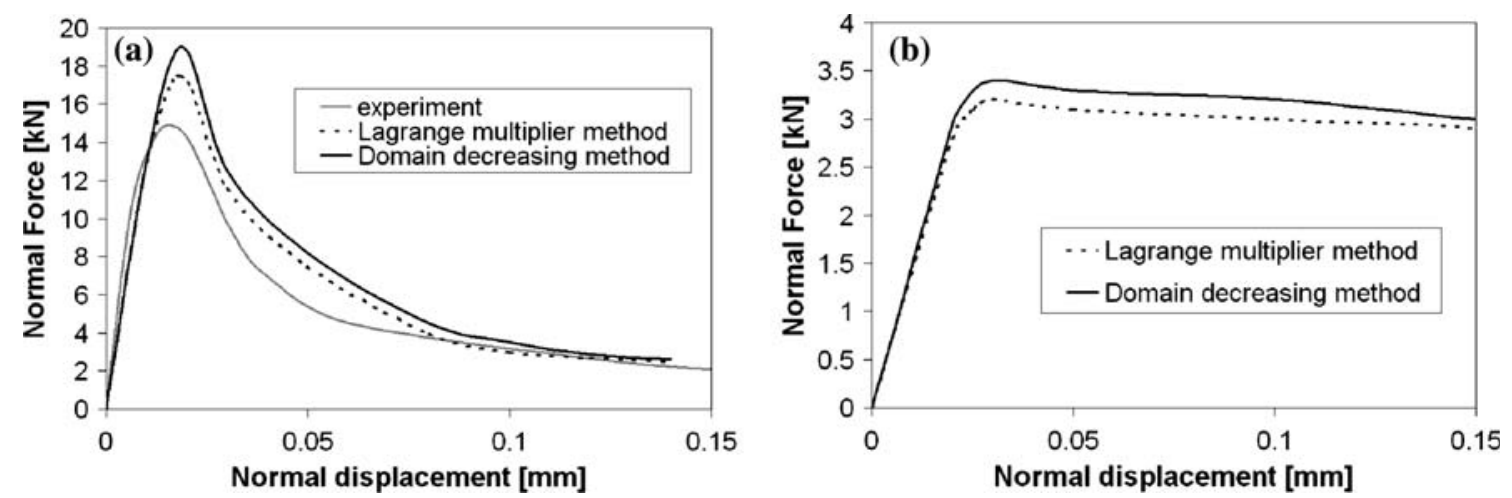

Fig. 14 The load deflection curve of the double edge-notch specimen for a load reversion of $P_{\mathrm{s}}=5 \mathrm{kN}$ and $\mathbf{b}$ load reversion of $P_{\mathrm{S}}=27.5 \mathrm{kN}$

two discretizations, one with approximately 4,000 particles and the second one with 14,000 particles. The crack had an angle of $23^{\circ}$ against the $y$-axis for the first computation (see Fig. 21a), which matched the experimental data pretty well; see John and Shah [24]. The results for the different refinements were almost identical.
Finally, we ran the problem with the notch located at $\gamma=0.50$ far away from the left support. We used 4,000 and 14,000 particles similar to the last example. The final crack pattern is shown in Fig. 22 and is very close to the experimental one; see John and Shah [24]. Only one crack occurs that is travelling from the end of the notch. A similar result was shown in $\mathrm{Zi}$ et al. [52], also. 


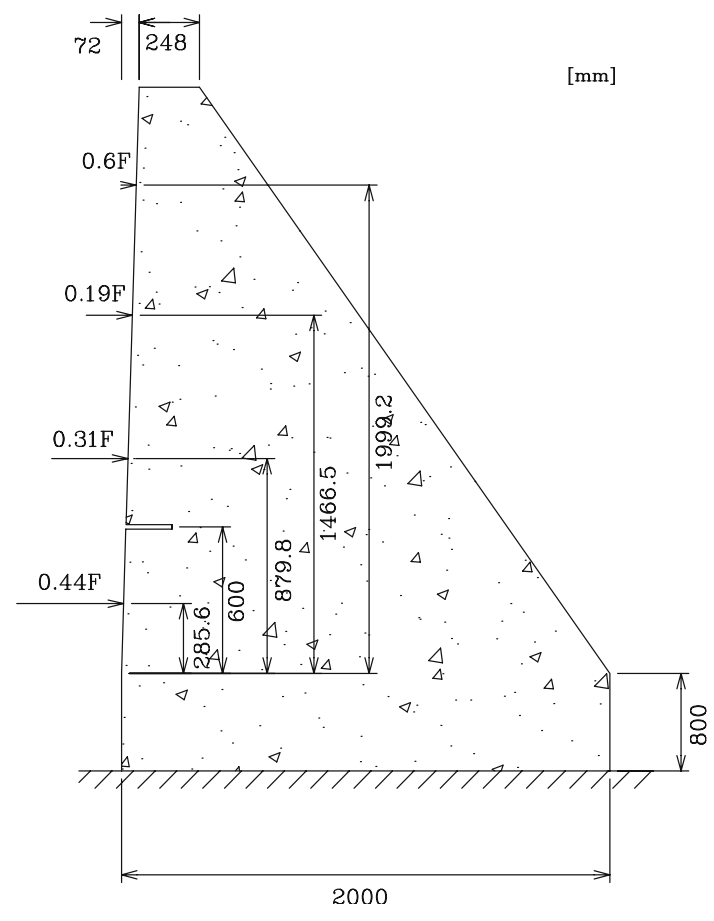

Fig. 15 A test model of concrete gravity dam by Carpinteri et al. [15]

\section{Conclusion}

1. We developed a new meshfree method for cohesive cracks. In this new method, the entire crack is enriched by the sign function, i.e. the branch enrichment is removed from the discontinuous displacement field. The shape function of the domain of influence containing the crack tip is modified so that the crack tip is always positioned on the edge $\sigma \quad[\mathrm{mm}]$

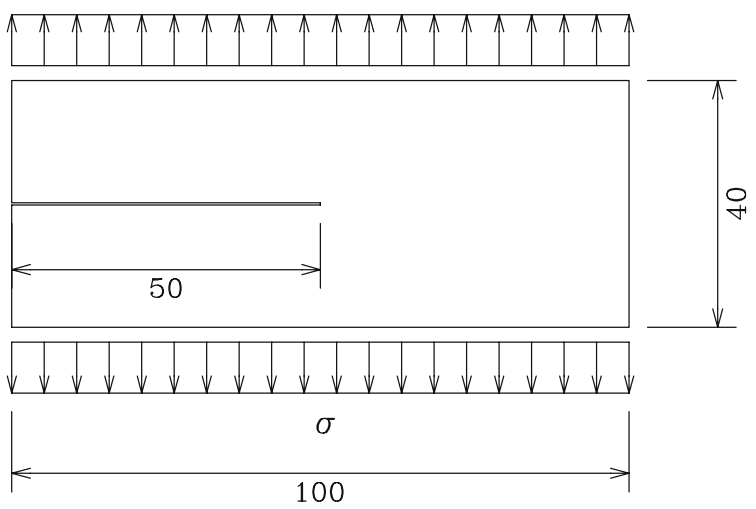

Fig. 17 A plate with an edge crack loaded by a uniform traction on the top and bottom edges

of the domain of influence. The modification is only for the discontinuous displacement field; the continuous displacement field is not changed.

2. We showed that the classical Lagrange multiplier can be used to remove the branch enrichment from the discontinuous displacement field. The crack is extended virtually beyond the crack tip so that the domains of influence containing the crack tip are completely cut by the crack. Then, the crack opening displacement along the virtual extension is enforced to vanish. The Lagrange multiplier is naturally discretized using the shape functions cut by the virtual extension. Therefore, a few additional unknowns should be introduced for each crack tip.

3. The developed methods are applied to well-known static and dynamic problems. It is demonstrated that the methods can reproduce the experimen-
Fig. 16 The development of cracks in dam by the two different methods

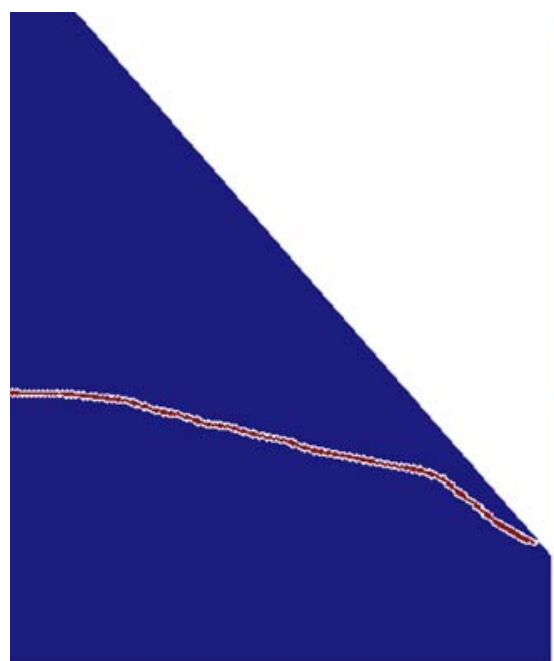

(a) 12,000 particles

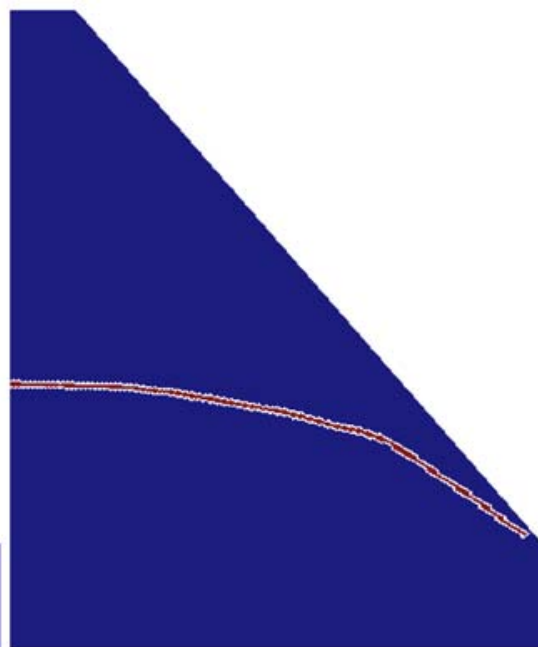

(b) 25,000 particles 
Fig. 18 The cracking patterns of the plate with an edge crack at different time steps for the two different methods

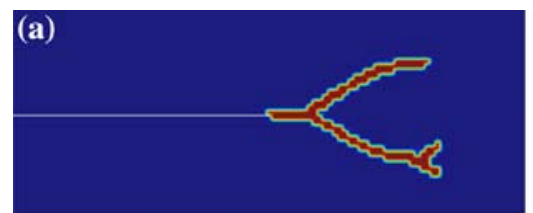

Domain-decrease method with 4,000 particles at time $0.045 \mathrm{~ms}$

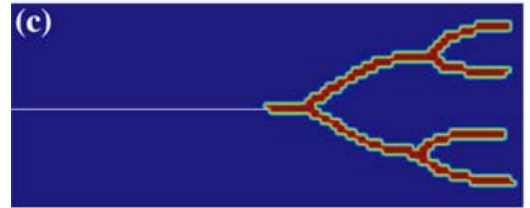

Domain-decrease method with 4,000 particles at time $0.062 \mathrm{~ms}$

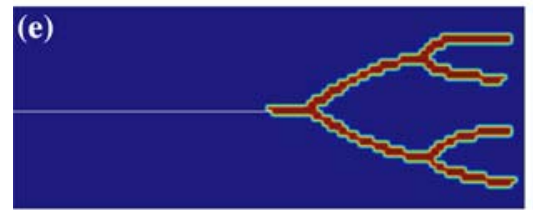

Lagrange multiplier method with 4,000 particles at time $0.062 \mathrm{~ms}$

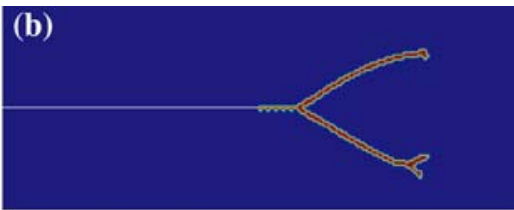

Domain-decrease method with 16,000 particles at time $0.045 \mathrm{~ms}$

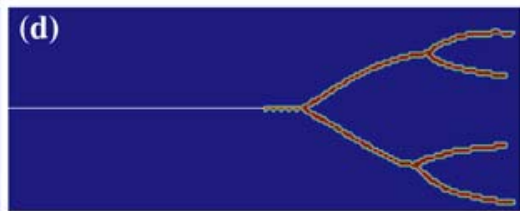

Domain-decrease method with 16,000 particles at time $0.062 \mathrm{~ms}$

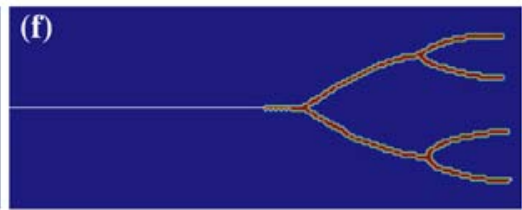

Lagrange multiplier method with 16,000 particles at time $0.062 \mathrm{~ms}$

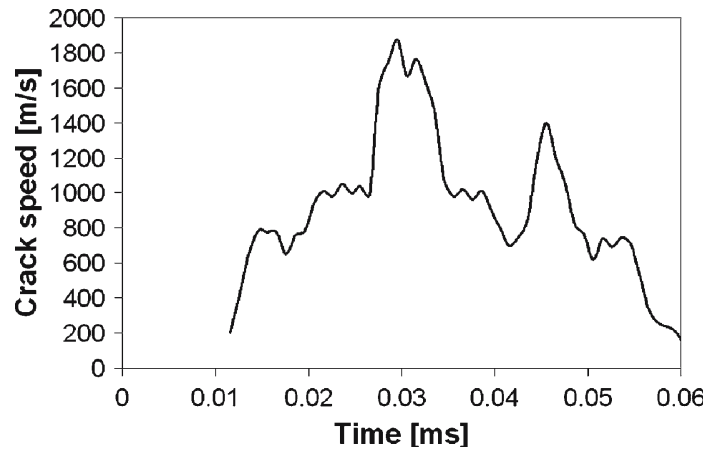

Fig. 19 The crack speed time history for the crack branching problem

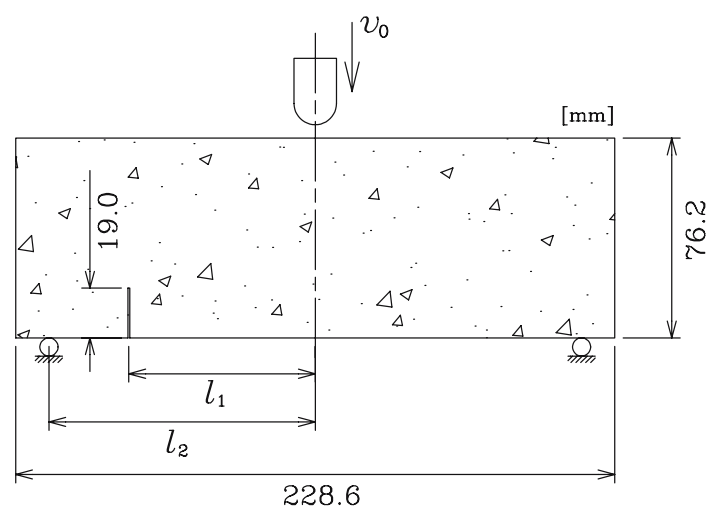

Fig. 20 The test for a mixed-mode dynamic fracture of concrete beam by John and Shah [24]; $l_{2}=101.6 \mathrm{~mm}$
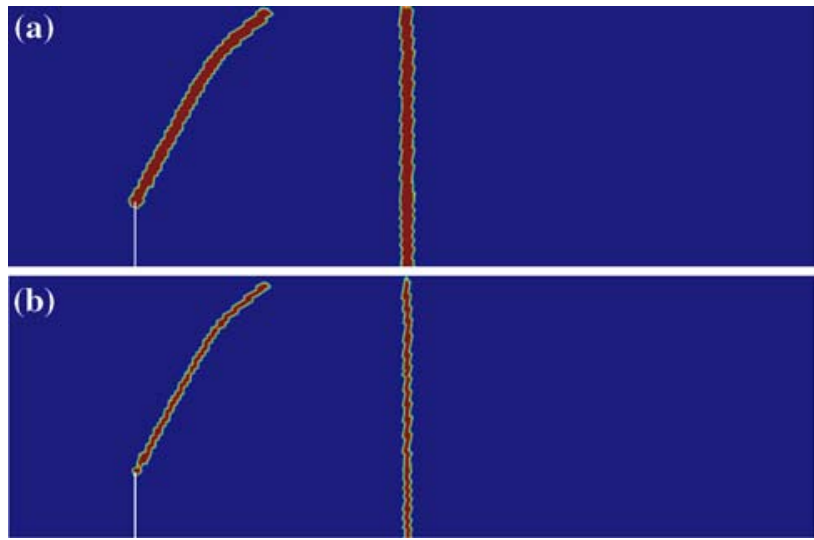

Fig. 21 The crack pattern of the John and Shah beam under impact loading for a location of the notch at $\gamma=0.77$; a 4,000 particles b 14,000 particles

tal results and the simulations by other researchers very well. The results of the two proposed methods produced almost same results. If the domain of influence is decreased, the results tend to be a little softer since the crack appears to be a little shorter. However the global response is barely influence by this effect.

4. The loss of hyperbolicity is used to determine the speed of crack propagation and the direction. The speed of crack propagation calculated from the loss of hyperbolicity is less than the Rayleigh wave speed and seems acceptable theoretically. 

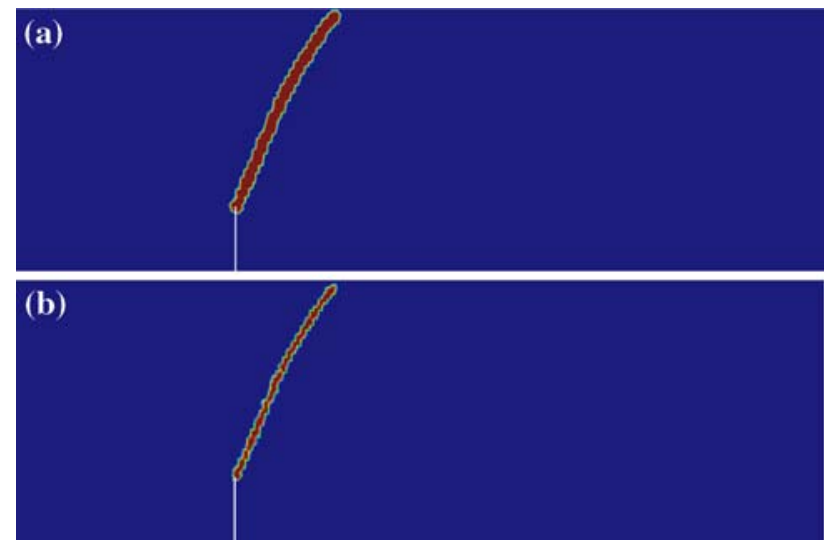

Fig. 22 The crack pattern of the John and Shah beam under impact loading for a location of the notch: $\gamma=0.50$; a 4,000 particles and $\mathbf{b}$ 14,000 particles

Acknowledgements We would like to thank the Agency of Defense Development (ADD) and the Korea Institute of Construction \& Transportation Technology Evaluation and Planning (KICTTEP) for their grants ADD-06-05-06 and 05-CTRM-D0403 , respectively.

\section{References}

1. Arrea M, Ingraffea AR (1982) Mixed-mode crack propagation in mortar and concrete. Technical Report 81-13, Department of Structural Engineering, Cornell University, Ithaka, NY

2. Bažant ZP, Belytschko T (1985) Wave propagation in a strainsoftening bar: Exact solution. J Eng Mech ASCE 111(3):381389

3. Bažant ZP, Caner FC (2005) Microplane model M5 with kinematic and static constraints for concrete fracture and anelasticity. I: Theory. J Eng Mech ASCE 131(1):31-40

4. Bažant ZP, Zi G (2003) Asymptotic stress intensity factor density profiles for smeared-tip method for cohesive fracture. Int J Fract 119:145-159

5. Belytschko T, Black T (1999) Elastic crack growth in finite elements with minimal remeshing. Int $\mathrm{J}$ Numer Meth Eng 45(5):601-620

6. Belytschko T, Chen H, Xu J, Zi G (2003) Dynamic crack propagation based on loss of hyperbolicity with a new discontinuous enrichment. Int J Numer Meth Eng 58(12):1873-1905

7. Belytschko T, Fleming M (1999) Smoothing, enrichment and contact in the element-free Galerkin method. Comput Struct 71(2):173-195

8. Belytschko T, Krongauz Y, Organ D, Fleming M, Krysl P (1996) Meshless methods: an overview and recent developments. Comput Methods Appl Mech Eng 139:3-47

9. Belytschko T, Liu WK, Moran B (2000) Nonlinear finite elements for continua and structures. Wiley New York

10. Belytschko T, Lu YY, Gu L (1994) Element-free Galerkin methods. Int J Numer Methods Eng 37(2):229-256

11. Belytschko T, Lu YY, Gu, L (1995) Crack-propagation by element-free Galerkin methods. Eng Fract Mech 51(2):295315

12. Belytschko T, Moës, N, Usui S, Parimi C (2001) Arbitrary discontinuities in finite elements. Int J Numer Methods Eng 50(4):993-1013
13. Belytschko T, Organ D, Gerlach C (2000) Element-free Galerkin methods for dynamic fracture in concrete. Comput Methods Appl Mech Eng 187(3-4):385-399

14. Camacho G, Ortiz M (1996) Computational modelling of impact damage in brittle materials. Int J Solids Struct 33:28992938

15. Carpinteri A, Valente S, Ferrara G, Imperato L (1992) Fracture mechanics of concrete structures, Elsevier New York, 351-360

16. Chessa J, Wang H, Belytschko T (2003) On the construction of blending elements for local partition of unity enriched finite elements. Int J Numer Methods Eng 57(7):1015-1038

17. Daux C, Moës N, Dolbow J, Sukumar N, Belytschko T (2000) Arbitrary branched and intersecting cracks with the extended finite element method. Int J Numer Methods Eng 48:17411760

18. Dolbow, JE, Devan A (2003) Enrichment of enhanced assumed strain approximations for representing strong discontinuities: addressing volumetric incompressibility and the discontinuous patch test. Int $\mathbf{J}$ Numer Methods Eng pages 59(1):47-67

19. Falk ML, Needleman A, Rice, JR (2001) A critical evaluation of cohesive zone models of dynamic fracture. J Phys IV, 11(PR5):43-50

20. FIB (1999) Structural concrete: Textbook on behaviour, design and performance updated knowledge of the CEB/FIP model code 1990. Vol. 1: Introduction - design process - materials International federation for structural concrete (fib), Lausanne, Switzerland

21. Fineberg J, Sharon E, Cohen G (2003) Crack front waves in dynamic fracture. Int J Fract 121(1-2):55-69

22. Fleming M, Chu YA, Moran B, Belytschko T (1997) Enriched element-free Galerkin methods for crack tip fields. Int $\mathrm{J}$ Numer Methods Eng 40:1483-1504

23. Hao S, Liu WK, Qian D (2000) Localization-induced band and cohesive model. J Appl Mech ASME 67:803-812

24. John R, Shah SP (1990) Mixed mode fracture of concrete subjected to impact loading. J Struct Eng ASCE 116(3): 585-602

25. Laborde P, Pommier J, Renard Y, Salaün M (2005) Highorder extended finite element method for cracked domains. Int J Numer Methods Eng 64(3):354-381

26. Lemaitre J, Chaboche J-L (1990) Mechanics of solid materials. Cambridge University Press, Cambridge ISBN 0521 328535

27. Li S, Liu WK (2004) Meshfree and particle methods. Springer, Berlin Heidelberg New York

28. Liu WK, Hao S, Belytschko T, Li S, Chang CT (2000) Multiscale methods. Int J Numer Methods Eng 47(7):1343-1361

29. Liu WK, Jun S, Zhang YF (1995) Reproducing kernel particle methods. Int J Numer Methods Eng 20:1081-1106

30. Melenk JM, Babuška I (1996) The partition of unity finite element method: Basic theory and application. Comput Methods Appl Mech Eng 139(1-4):289-314

31. Moës N, Belytschko T (2002) Extended finite element method for cohesive crack growth. Eng Fract Mech 69(7):813-833

32. Moës N, Dolbow J, Belytschko T (1999) A finite element method for crack growth without remeshing. Int $\mathrm{J}$ Numer Methods Eng 46(1):131-150

33. Nooru-Mohamed MB (1992) Mixed-mode fracture of concrete: An experimental approach. PhD thesis, Delft University of Technology

34. Oliver J, Huespe AE, Samaniego E, Chaves EMV (2004) Continuum approach to the numerical simulation of material failure in concrete. Int J Numer Anal Methods Geomech 28(7-8):609-632 
35. Ortiz M, Pandolfi A (1999) Finite-deformation irreversible cohesive elements for three-dimensional crack-propagation analysis. Int J Numer Methods Eng 44(9):1267-1282

36. Pandolfi A, Guduru PR, Ortiz M (2000) Three dimensional cohesive-element analysis and experiments of dynamic fracture in C300 steel. Int J Solids Struct 37:3733-3760

37. Patzák B, Jirásek M (2004) Adaptive resolution of localized damage in quasi-brittle materials. J Eng Mech ASCE 130(6):720-732

38. Rabczuk T, Belytschko T (2004) Cracking paricles: A simplified meshfree method for arbitrary evolving cracks. Int $\mathrm{J}$ Numer Methods Eng 61:2316-2343

39. Rabczuk T, Belytschko T (2006) A three dimensional large deformation meshfree method for arbitrary evolving cracks. Comput Methods Appl Mech Eng (in press)

40. Rabczuk T, Zi G (2006) A meshfree method based on the local partition of unity for cohesive cracks. Comput Mech (in press)

41. Ravi-Chandar K (1998) Dynamic fracture of nominally brittle materials. Int J Fract 90(1-2):83-102

42. Sethian JA (1999) Level sets methods \& fast marching methods: Evolving interfaces in computational geometry, fluid mechanics, computer vision and materials science. Cambridge University Press, Cambridge

43. Sharon E, Fineberg J (1996) Microbranching instability and the dynamic fracture of brittle materials. Phys Rev B 54(10):7128-7139

44. Shi Z (2004) Numerical analysis of mixed-mode fracture in concrete using extended fictitious crack model. J Struct Eng ASCE 130(11):1738-1747
45. Simonsen BC, Li S (2004) Meshfree modeling of ductile fracture. Int J Numer Methods Eng 60:1425-1450

46. Stazi F, Budyn E, Chessa J, Belytschko T (2003) XFEM for fracture mechanics with quadratic elements. Comput Mech $31: 38-48$

47. Strouboulis T, Copps K, Babuška I (2000) The generalized finite element method: An example of its implementation and illustration of its performance. Int J Numer Methods Eng 47(8):1401-1417

48. Ventura G, Budyn E, Belytschko T (2003) Vector level set for description of propagating cracks in finite elements. Int $\mathbf{J}$ Numer Methods Eng 58(10):1571-1592

49. Ventura G, Xu JX, Belytschko T (2002) A vector level set method and new discontinuity approximations for crack growth by EFG. Int J Numer Methods Eng 54(6):923-944

50. Xu X-P, Needleman A (1994) Numerical simulation of fast crack growth in brittle solids. J Mech Phys Solids 42(9):13971434

51. Zi G, Belytschko T (2003) New crack-tip elements for XFEM and applications to cohesive cracks. Int J Numer Methods Eng 57:2221-2240

52. Zi G, Chen H, Xu J, Belytschko T (2005) The extended finite element method for dynamic fractures. Shock Vibration 12(1):9-23

53. Zi G, Song J-H, Budyn E, Lee S-H, Belytschko T (2004) A method for growing multiple cracks without remeshing and its application to fatigue crack growth. Modell Simul Materials Sci Eng 12:901-915 